\title{
Microneedle-mediated transdermal transport of selected triptans: frovatriptan succinate monohydrate and rizatriptan benzoate
}

\author{
Gagandeep K Malhi ${ }^{1}$, Kevin B Ita ${ }^{1 *}$, Matthew J Morra ${ }^{2}$ and Inna E Popova ${ }^{2}$ \\ ${ }^{1}$ College of Pharmacy, Touro University, Mare Island-Vallejo, California, 94592, USA \\ ${ }^{2}$ Department of Soil and Water Systems, University of Idaho, Moscow, Idaho, 83844-2340, USA
}

\begin{abstract}
Migraine is a debilitating neurological disorder which is characterized by recurring pulsatile pain on one or both sides of the head. Triptans are used for the management of migraine. These therapeutic agents act by activating the serotonin 5-HT signaling pathway. In this project, our aim was to develop transdermal drug delivery systems for two medications [frovatriptan succinate monohydrate and rizatriptan benzoate] commonly used for the management of migraine. The rationale is that transdermal drug delivery systems are capable in increasing the bioavailability of medications. In this project, we wanted to find out if microneedle rollers can significantly increase the percutaneous transport of frovatriptan succinate monohydrate and rizatriptan benzoate across pig ear skin in vitro. The flux value for microneedle-assisted drug permeation of frovatriptan across pig ear skin was $25.7 \pm 2.5 \mu \mathrm{g} / \mathrm{cm}^{2} / \mathrm{h}$ in comparison to passive transdermal flux which was $13.46 \pm 1.3$ $\mu \mathrm{g} / \mathrm{cm}^{2} / \mathrm{h}$. The mean passive flux of rizatriptan was $20.2 \pm 5.0 \mu \mathrm{g} / \mathrm{cm}^{2} / \mathrm{h}$ as compared to microneedle-mediated flux [51.8 $\pm 9.3 \mu \mathrm{g} / \mathrm{cm}^{2} / \mathrm{h}$ ]. Student's t-test determined that there was a statistically significant difference $[\mathrm{p}<0.05]$ between the flux values of microneedle-mediated transport of frovatriptan succinate monohydrate and rizatriptan benzoate across pig ear skin in comparison with passive diffusion.
\end{abstract}

\section{Introduction}

Migraine is a common neurological disorder which is characterized by recurring pulsatile pain on one or both sides of the head [1]. In the United States, there are nearly 37 million patients who suffer from migraine [2,3]. Migraine attacks can last from 4-72 hours, aggravated by movement and may cause individuals to often experience a significant decline in their routine physical activities [4]. The episodic disorder is a significant socioeconomic problem because migraine attacks are ranked sixth in the cause of years of life lost in individuals due to physical or mental impairment in the universal population and also rank third for years of life lost in individuals $<50$ years old [5-7]. Symptoms of this disorder present as moderate-to-severe headaches, nausea, emesis, dizziness, blurred vision, and sensitivity to light, sound, and touch [1]. There are two main types of migraine, the common migraine and the classic migraine. Classic migraine is accompanied by visual disturbances (aura) [8]. The etiology of migraine is still unknown; however, it is postulated that the disorder involves abnormal activation of the trigeminal vascular system (TGVS) [9]. Several researchers have pointed out that migraine is associated with the imbalance in inhibitory and excitatory factors affecting brain function [10]. Although there is no cure for migraine, various drug treatments are available to alleviate symptoms [11].

There are numerous medications used in management of migraine, especially when patients complain of frequent attacks $[12,13]$. Frequencies fluctuate back and forth from a moderate to severe pattern and each year about $3 \%$ of individuals who suffer from episodic migraine attacks report headaches occurring more than 15 days/month [7]. Pharmacotherapy is aimed at eliminating attacks and providing prompt relief from pain. Triptans are commonly used as medications for the management of migraine attacks [14]. Serotonin (5-HT) is mainly found in three main cell types-(a) serotonergic neurons in the CNS and in the intestinal myenteric plexus, (b) enterochromaffin cells in the mucosa of the gastrointestinal tract and (c) in blood platelets [15]. Three distinct pharmacological actions have been implicated in the antimigraine effect of the triptans [16] - (1) stimulation of presynaptic 5-HT1D receptor, which serves to inhibit both dural vasodilation and inflammation (2) direct inhibition of trigeminal nuclei cell excitability via $5-\mathrm{HT} 1 \mathrm{~B} / 1 \mathrm{D}$ receptor agonism in the brainstem and (3) vasoconstriction of meningeal, dural, or cerebral vessels as a result of vascular 5-HT1B receptor agonism $[1,17]$. $5-\mathrm{HT}$ is a key mediator in pathogenesis of migraine. It has been suggested that migraine is associated with a low level of serotonin which activates the trigeminovascular nociceptive pathway, altering 5-HT neurotransmission and manifesting a migraine headache [18]. Consequently, serotonin agonists are used to treat migraine because they narrow cranial blood vessels [19-21]. (19-21). The successful use of the first generation triptan, sumatriptan, has led to the development of other compounds with much improved pharmacokinetic and pharmacodynamic properties, efficacy, and safety [22]. Second generation triptans are more lipophilic and capable of penetrating the blood brain barrier, thereby reaching their site of action readily [22].

*Correspondence to: Ita KB, College of Pharmacy, Touro University, Mare Island-Vallejo, California, 94592, USA, Tel: +1 [707] 638-5994; Email: kevin. ita@tu.edu

Key words: migraine, transdermal drug delivery, microneedle roller, frovatriptan succinate monohydrate, rizatriptan benzoate

Received: July 10, 2018; Accepted: July 19, 2018; Published: July 22, 2018 
Frovatriptan succinate monohydrate (FSM) is a second-generation triptan used for the management of acute migraine (with or without aura) [23]. It has a molecular weight of $379.41 \mathrm{~g} / \mathrm{mol}$ [24]. The precise mechanism of action of frovatriptan is unknown, but is thought to be associated with agonism at serotonin 5-HT1B and 5-HT1D receptors, resulting in inhibition of intracranial and extracerebral artery vasodilation [8]. FSM has the highest 5-HT1B potency in the triptan class. With a long terminal elimination half-life of 26 hours, FSM has greater clinical consistency and sustained effect with lower risk of recurrence $[23,25,26]$.

Rizatriptan benzoate (RB) is a selective agonist of serotonin and stimulates $5-\mathrm{HT} 1 \mathrm{~B} / 1 \mathrm{D}$ receptor [27]. It has a molecular weight of $391.47 \mathrm{~g} / \mathrm{mol}$ and a weak affinity for subtypes 5-HT1A, 5-HT5A, and 5-HT7 [27]. Like FSM, RB binds selectively with high affinity for $5-\mathrm{HT}$ receptor subtypes $1 \mathrm{~B} / 1 \mathrm{D}$. RB acts by stimulating the presynaptic $5-\mathrm{HT} 1 \mathrm{~B} / 1 \mathrm{D}$ receptor, inhibiting dural vasodilation, inflammation, and reducing the excitability of trigeminal nuclei cell $[28,29]$. This action aids in the prevented release of nociceptive neuropeptides from presynaptic nerves and reduced trigeminal pain signal transmission [30].

Triptans are commonly administered orally as tablets. However, there are many limitations with the oral route of drug administration such as erratic absorption, gastric irritation, enzymatic degradation and pre-systemic metabolism [31]. In addition, patients may experience vomiting and nausea symptoms [32]. Currently there are 35 approved transdermal products [33] with about 21 active pharmaceutical ingredients.

Transdermal drug delivery (TDD) refers to the application of a drug to the skin for systemic effect [34]. The drug initially penetrates through the stratum corneum [SC] and then passes through the viable epidermis and dermis [35]. When the drug reaches the dermis, it becomes available for systemic absorption through blood vessels located in the dermis $[32,36,37]$. TDD has many advantages over the oral and injection routes such as drug delivery at a controlled rate, non-invasiveness, and avoidance of needle sticks, controlled high blood concentrations, and simple to use [38-40]. Furthermore, enhanced bioavailability and a more uniform drug plasma level may lead to increased efficacy and a reduction in adverse events [32]. TDD improves patient compliance due to the reduction of dosing frequencies and is also more amenable to self-administration [41]. Despite the advantages, TDD faces a challenge against the skin barrier due to the unique structure of the human skin.

The skin is the largest organ in the body and provides protection for internal organs $[42,43]$. The human skin comprises the epidermis, dermis and the subcutaneous layer. The stratum corneum is the outer most layer of the skin with an average thickness of $13-20 \mu \mathrm{m}$ $[44,45]$. The epidermis consists of keratinocytes with proteins and tight junctions [46]. With an aqueous environment, diffusion rate of lipophilic compounds may be affected. To penetrate the skin, drug molecules must cross the lipid-rich stratum corneum [32,34]. Percutaneous transport of drug molecules through the skin can be also achieved by diffusion through hair follicles and sweat glands [35]. There are numerous methods for enhancing drug delivery through the skin [47]. These include chemical enhancers, and physical enhancers such as sonophoresis, iontophoresis and microneedles [29,48].

Microneedles [MNs] are micron-sized needles used for transdermal drug delivery [49]. These devices create microchannels in the skin thereby facilitating drug transport $[49,50]$. MNs can be categorized into five types. These are solid, hollow, coated, dissolving and hydrogel- forming [51,52]. MNs are painless, minimally invasive and are easily disposable [51]. The use of microneedles does not always guarantee that there will be an increase in the transdermal diffusion rate. For example, Vitorino and co-workers showed that the use of a $500 \mu \mathrm{m}$ microneedle roller [Dermaroller ${ }^{\otimes}$ ] had little or no effect on the drug transdermal delivery of olanzapine and simvastatin [53]. The interactions between the skin, drugs, and microneedle devices are complex and can only be determined experimentally.

The aim of our research was to investigate the effect of solid microneedle rollers on the transport of two antimigraine drugs, frovatriptan succinate monohydrate and rizatriptan benzoate, across pig ear skin in vitro.

\section{Materials}

The microneedle rollers were purchased from Pearl Enterprises LLC [Lakewood, New Jersey, USA]. RB, FSM, ethanol, and $0.1 \mathrm{M}$ phosphate-buffered saline (PBS), were purchased from Sigma Aldrich [Saint Louis, Missouri, USA]. Distilled ionized (DI) water was obtained from NanoPure Infinity Ultrapure [Barnstead, Dubuque, Iowa, USA]. High-vacuum silicone grease gel (Dow Corning ${ }^{\circledR}$ ) was also purchased from Sigma Aldrich (Saint Louis, Missouri, USA).

\section{Methods}

\section{Pig ear skin preparation}

Pig ears were bought from Pel-Freez Biologicals (Rogers, Arkansas, USA). Each pig ear was first thawed to room temperature and shaved with a hair clipper. An electric dermatome (Nouvag) was used to prepare split thickness skin samples. The thickness of each pig ear skin sample was measured with Mitutoyo micrometer (Mitutoyo, Tokyo, Japan) with an average of $542 \mu \mathrm{m}$. Skin membranes were then wrapped in aluminum foil and stored at $-20{ }^{\circ} \mathrm{C}$ for experiments and LC-MS analysis. Laboratory experiments were authorized by the Institutional Biosafety Committee (IBC) and the Institutional Animal Care and Use Committee (IACUC) of Touro University California.

\section{Visualization of microchannel by Confocal Microscopy}

Confocal microscopy (CFM) was used to visualize microchannels created using solid microneedles on porcine ear skin. CFM was carried out at the Laura Van Winkle Laboratory at the University of California, Davis. Pig ear skin samples were initially treated with a microneedle roller by applying pressure in vertical and horizontal strokes. Next, the pre-treated skin samples were stained with Alexa Fluor dye 488 from Sigma Aldrich followed by a rinse with DI water to remove superfluous dye. For the control, intact porcine ear skin samples (without microneedle pretreatment) were similarly stained with Alexa Fluor dye 488 and rinsed as well. Images of the treated and untreated skin samples were obtained using the Leica DFC-295 (Davis, California, USA).

\section{Diffusion Studies}

In vitro diffusion studies were carried out using a Franz diffusion cell system consisting of 6 cells. Each individual cell was made up of a donor compartment, receptor compartment, a sampling port and a magnetic stirrer, maintained at $37^{\circ} \mathrm{C}$ to mimic the human body temperature. The acceptor compartment had a volume capacity of 12 $\mathrm{mL}$ filled with PBS and diffusion surface area of $1.77 \mathrm{~cm}^{2}$.

Porcine skin samples were treated with a solid microneedle roller to create pores and microchannels. This was done so by applying pressure ( $\sim \mathrm{kg}$ for 60 seconds) using the microneedle roller against 
the skin membrane, five times in each direction, per application. This effort ensures that the skin samples will have a greater number of microconduits increasing the efficiency of TDD. After the application, both pretreated and untreated porcine skin samples were placed between the donor compartment and the receptor compartment, sandwiching the skin membrane. A high-vacuum grease gel (Dow Corning $^{\bullet}$, Midland, Massachusetts, USA) and a metal clamp were used to seal the system. During each experiment, the donor compartment would consist of $1 \mathrm{~mL}$ of either FSM or RB loaded over all 6 skin samples. The top of the donor compartment and the sampling port were covered with parafilm and aluminum foil to ensure that there is no contamination or evaporation of the liquid compound.

Diffusion experiments of FSM and RB were repeated 6 times $(n=6)$. The experiment was designed to last for a 12-hour period and $1 \mathrm{~mL}$ samples were taken every 2 hours from the sampling port of each cell and then carefully put into glass vials (Agilent Santa Clara, California, USA) and stored at $-8^{\circ} \mathrm{C}$ for LC-MS analysis. The cumulative amount of FSM and RB which permeated skin samples was plotted against time [0-12 hours]. Transdermal flux values were calculated from the linear portion of the cumulative amount versus time curves.

\section{Liquid Chromatography-Mass Spectrometry (LC-MS) Conditions}

High performance LC-MS analysis and diode array detection (DAD) of FSM and RB was performed using Agilent 1200 Series system. Time-of-flight mass spectrometry (TOF-MS) and Agilent 6230 Ion Trap detector (Agilent, Santa Clara, California, USA) were used. Chromatographic separation of FSM and RB was preformed using Zorbax Eclipse Plus $\mathrm{C}_{18}$ [100 mm x $2.1 \mathrm{~mm}, 3.5 \mu \mathrm{m}$ column], which was maintained at a temperature reading of $30^{\circ} \mathrm{C}$. The analytical column was protected by a guard column with a similar stationary phase (Agilent, Santa Clara, California, USA). The mobile phase was made up of $0.1 \%$ formic acid+water (solvent $A$ ) and $0.1 \%$ formic acid + acetonitrile (solvent $\mathrm{B})$.

A sample volume of $5 \mu \mathrm{L}$ was injected during the initial mobile phase B of the linear gradient program, starting from $3 \%$ to $20 \%$ in three minutes. Later the mobile phase B was increased from $20 \%$ to $100 \%$ in two minutes. An equilibration was conducted at mobile phase of $3 \%$ for a post-run time of five minutes after each sample was tested. To avoid ion suppression and MS contamination, flow rate was redirected during the first one minute of analysis, yielding a flow rate of $0.3 \mathrm{~mL} / \mathrm{min}^{-1}$. Calibration curves were prepared in fresh PBS.

In positive mode, MS data was collected using the electrospray ionization (ESI) potential and collision-induced dissociation potential. Absolute values were recorded at $3500 \mathrm{~V}$ and $175 \mathrm{~V}$, respectively. Parameters of MS data collected were set to the following: gas temperature of $350{ }^{\circ} \mathrm{C}, \mathrm{N}_{2}$ drying gas, flow rate of $10 \mathrm{~L} / \mathrm{min}^{-1}$, and lastly the nebulizer pressure was $2.4 \times 10^{5} \mathrm{~Pa}$. In centroid mode, analyses were conducted at an $\mathrm{m} / \mathrm{z}$ range of $90 \mathrm{amu}$ to $500 \mathrm{amu}$. Both compounds, FSM and RB, were quantified in positive ESI MS/MS and reconstructed ion mode yielding values of $244.13 \mathrm{~m} / \mathrm{z}$ and $270.16 \mathrm{~m} / \mathrm{z}$, respectively (Table 1).

\section{Data Analysis}

The average cumulative amount of drug diffusing through each porcine ear skin was plotted against time.Transdermal flux values were calculated from individual linear portions of the cumulative amount versus time curves. Six replicates $[n=6]$ were used in this study. The
Table 1. HPLC-MS Quantification Parameter

\begin{tabular}{|c|c|c|c|}
\hline Compound & $\begin{array}{c}\text { Retention time } \\
(\mathbf{m i n})\end{array}$ & $\begin{array}{c}\text { Quantification ion } \\
(\mathbf{m} / \mathbf{z})\end{array}$ & $\begin{array}{c}\text { Limit of detection } \\
(\mathbf{n g})\end{array}$ \\
\hline FSM & 1.3 & 244.13 & 0.1000 \\
\hline RB & 2.2 & 270.16 & 0.051 \\
\hline
\end{tabular}

corrected drug concentration for sampling effects was calculated with Equation 1(Hayton-Chen Equation) [54]:

$$
C_{n}^{1}=C_{n}\left(\frac{V_{T}}{V_{\tau}-V_{S}}\right)\left(\frac{C_{n-1}^{1}}{C_{n-1}}\right)
$$

Equation 1: Hayton-Chen equation

In Equation 1, represents the corrected concentration, $C_{n}$ represents the measured concentration in the $n$th sample, $V_{T}$ is the total volume in the acceptor compartment of the cell [12 $\mathrm{mL}], V_{s}$ is the volume of the sample taken from the acceptor compartment via sampling port $[1 \mathrm{~mL}]$, while represents the corrected concentration in $[n-1]^{\text {th }}$ sample, and $C_{n-1}$ represents the measured concentration in $[\mathrm{n}-1]^{\text {th }}$ sample.

\section{Statistical Analysis}

Statistical analysis was preformed using the GraphPad Prism (version 7) software. Student's t-test was carried out to determine the statistical significance. Mean of replicate measurements [n=6] with corresponding standard deviation was used to plot the graphs.

\section{Results}

\section{Characteristics of Microneedle Roller}

Solid microneedles leverage the advantages of minimal invasiveness, painlessness, ease of application, and avoidance of skin irritation [52]. A solid, stainless-steel microneedle roller was used in this study to deliver antimigraine agents FSM and RB across pig ear skin (Figure 1). Each roller was applied to the pig ear skin with force, vertically and horizontally, to ensure proper penetration of micronsized needles. The microneedle roller has a density of 192 needles and a length of $500 \mu \mathrm{m}$. Following the use of a microneedle roller, channels were imaged and visualized, as shown in Figure 2A and 2B.

\section{Visualization of Microchannels by Confocal Microscopy [CFM]}

Both treated and untreated porcine skin membranes were stained with Alexa Fluor dye 488 and later sectioned using the Leica CM1950 cyostat. Samples were then placed to rest on a clean microscope slide and observed under the CFM. Figure 2A shows an intact layer of skin in which the SC has not been disrupted, therefore showing no visualization of a microchannel. In contrary, Figure $2 \mathrm{~B}$ shows a sample of pig ear skin which has been pretreated with a microneedle roller. The microchannel depth is $265 \mu \mathrm{m}$.

\section{In Vitro Diffusion Studies}

The purpose of carrying out an in vitro diffusion study was to identify the effects and influence solid microneedles had on the percutaneous absorption of FSM and RB. This experiment used porcine ear skin samples as a representative of the human epidermal layer. In our experiments, the mean skin thickness of porcine skin samples were $462 \pm 0.15 \mu \mathrm{m}$.

The transdermal transport of FSM and RB was plotted as a cumulative amount versus the function of time in hours (Figures $3 \mathrm{~A}$ and $4 \mathrm{~A}$ ), respectively. It showed a steady increase after the use of microneedle rollers when compared to control [passive diffusion]. There is an increase in transdermal flux of FSM in vitro from $13.4 \pm 1.3$ 


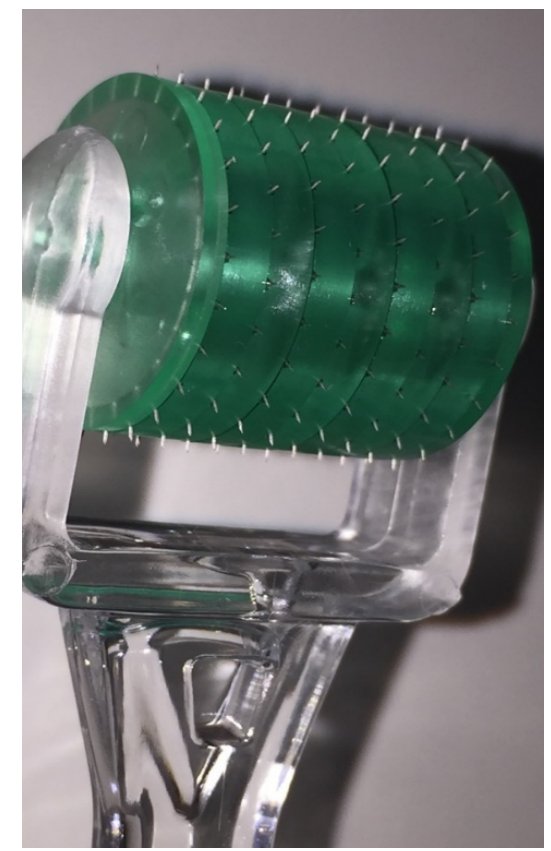

Figure 1. Solid stainless-steel microneedle roller (length $=500 \mu \mathrm{m}$; density=192 needles)

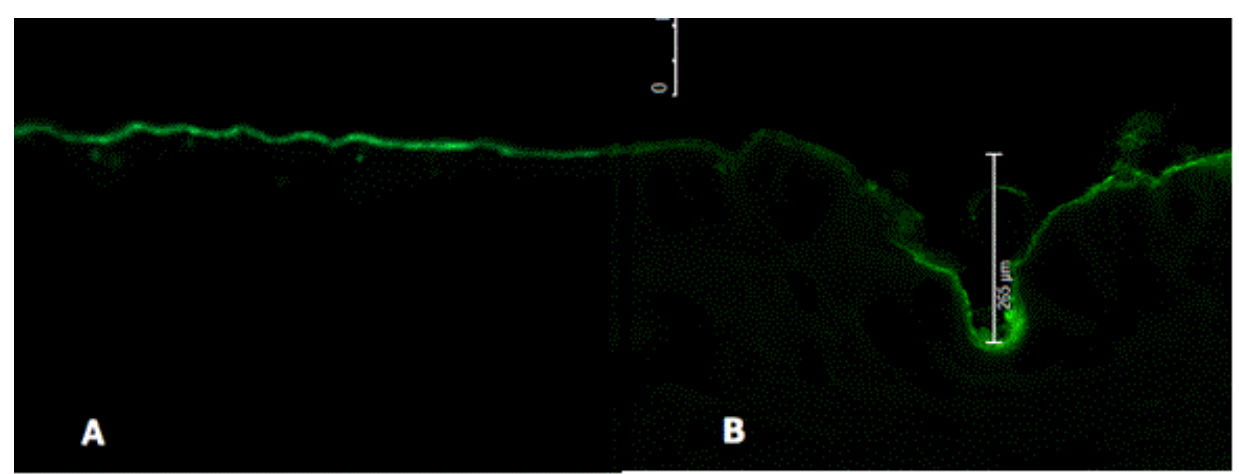

Figure 2. Visualization and characteristics depth of a single microchannel by CFM. (A) shows a sample of untreated porcine skin (control) and (B) displays a microneedle-treated porcine skin $($ depth $=265 \mu \mathrm{m})$

$\mu \mathrm{g} / \mathrm{cm}^{2} / \mathrm{h}$ across untreated porcine skin to $25.7 \pm 2.5 \mu \mathrm{g} / \mathrm{cm}^{2} / \mathrm{h}$ across microneedle-treated porcine skin, as shown in Figure 3B. RB showed a transdermal flux value of untreated porcine skin $\left(20.2 \pm 5.0 \mu \mathrm{g} / \mathrm{cm}^{2} / \mathrm{h}\right)$ compared to an enhanced flux value across microneedle-mediated porcine skin $\left(51.8 \pm 9.3 \mu \mathrm{g} / \mathrm{cm}^{2} / \mathrm{h}\right)$ (Figure $\left.4 \mathrm{~B}\right)$.

\section{Discussion}

The focus of our study was to test an alternative route of administration, transdermal drug delivery, for two anti-migraine agents (FSM and RB). We hypothesized that the use of solid microneedle rollers will increase transdermal flux values for FSM and RB. Our results indicated that there was a statistically significant difference in transdermal flux values of FSM and RB when microneedle rollers were used in comparison to passive diffusion.

The intended development of an alternative route of administration such as TDD was driven by an unmet medical need. Hoang et al. described the long-term AEs of using oral non-ergot dopamine agonists in the management of Parkinson's disease [54]. By using MNs, the authors investigated the influence of solid microneedles on the percutaneous transport of amantadine hydrochloride and pramipexole
dihydrochloride(54).Building on their rationale in developing transdermal formulations to yield stable plasma concentrations and better patient compliance [54], we studied the transcutaneous flux of FSM and RB. Transdermal drug administration is painless, and can lead to improved patient compliance [54] particularly in the elderly, adolescents, and patients who have difficulty with swallowing oral tablets [49]. Some of the issues associated with oral tablets and injections can be overcome by utilizing the transdermal route [49].

The human skin is the largest organ and constitutes a formidable defense against microorganisms and toxic chemicals [55]. The skin is made up of three layers: the epidermis, dermis, and the hypodermis. The SC is made up of corneocytes embedded in a lipid matrix $[55,56]$, however the SC poses a challenge to drug delivery [49,54]. Before absorption in the dermis, a drug molecule has several barriers to overcome [46]. In our study, we used solid stainless-steel microneedle rollers with a length of $500 \mu \mathrm{m}$ and a density of 192 needles to create microchannels in the SC. Confocal microscopy images confirmed that solid microneedle roller did in fact successfully break intact porcine skin and create microchannels (average $265 \mu \mathrm{m}$ ), allowing percutaneous penetration of FSM and RB. 

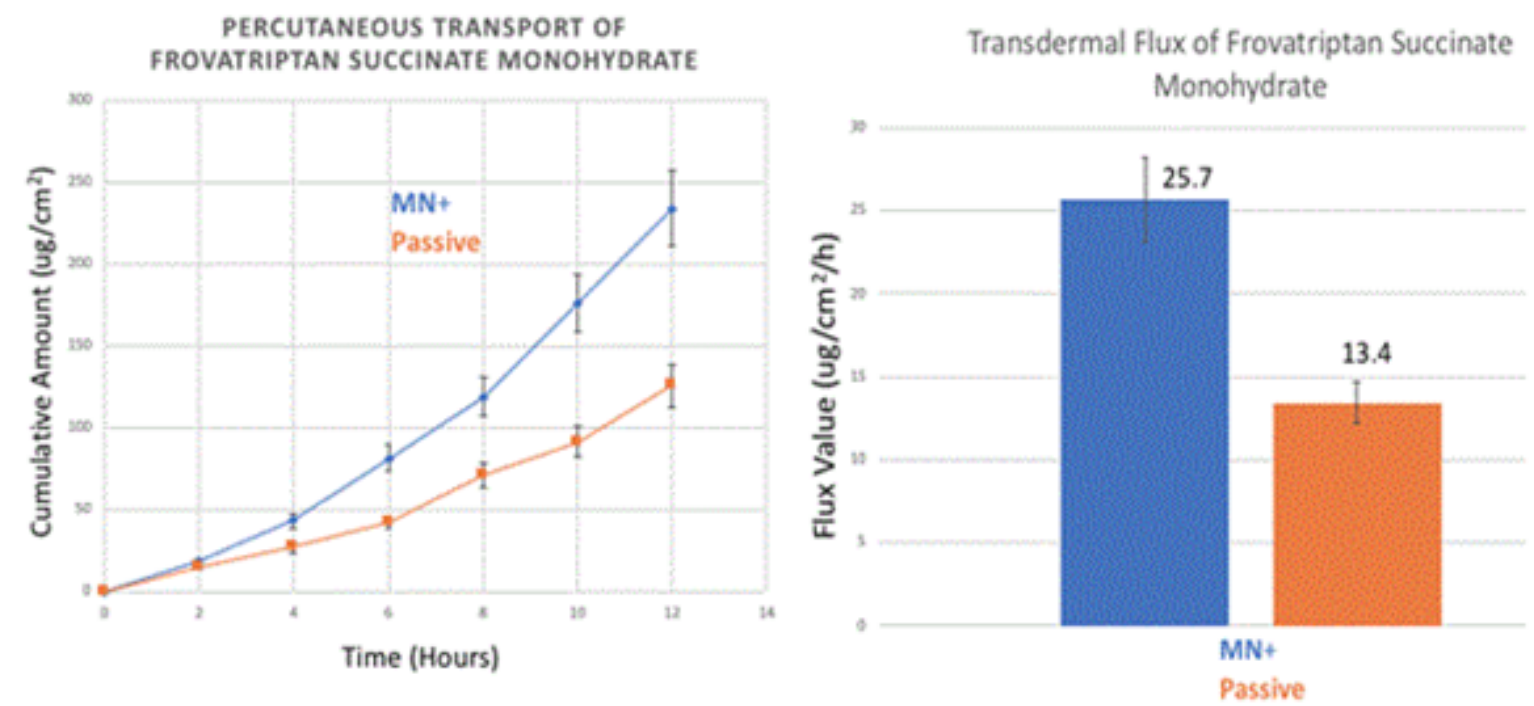

Figure 3. In vitro transdermal permeation of passive and microneedle-mediated FSM after solid microneedle roller application across porcine ear skin. (A) represents the cumulative amount versus time curves while (B) represents transdermal flux values taken from the slope of the linear portion of the cumulative amount versus time
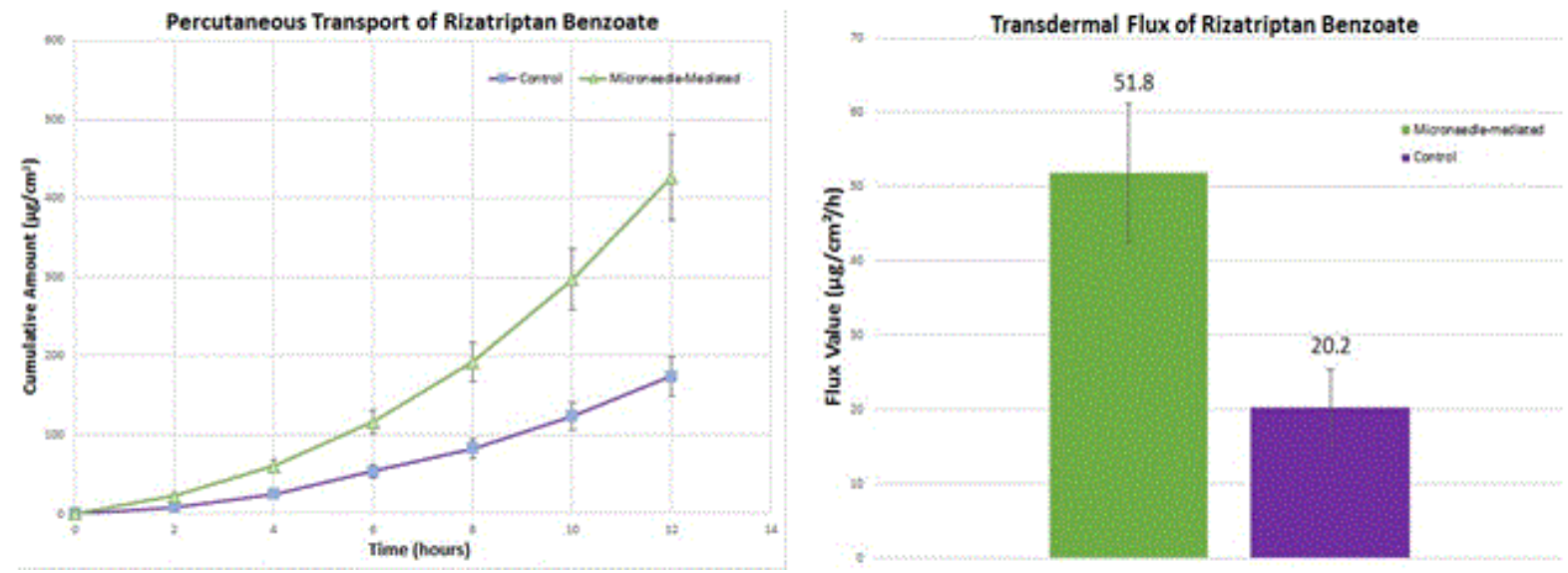

Figure 4. In vitro transdermal permeation of passive and microneedle-mediated RB after solid microneedle roller application across porcine ear skin. (A) represents the cumulative amount versus time curves while (B) represents transdermal flux values taken from the slope of the linear portion of the cumulative amount versus time.

Paracellular or intercellular routes between the corneocytes are identified as a primary transport pathway for lipophilic drug molecules $[33,52]$. This route requires the drug to diffuse through bilayers of ceramides made of free fatty acids, esters, and cholesterol. Depending on the anatomical site, rate of diffusion may be affected by differences in corneocytes sizes.

Diffusion rate also can depend on multiple factors such as molecular weight, kinetic energy of the molecule, concentration gradient, use of physical or chemical enhancers, and distance from the $\mathrm{SC}$ to the main bloodstream. Current physiochemical requirements for transdermal drug candidates for passive diffusion is that the molecules must be potent, highly lipophilic, low in molecular weight $[<500 \mathrm{Da}]$, low melting point $\left(<150^{\circ} \mathrm{C}\right)$, sufficient $\mathrm{pH}$ (between 6 to 7.4$)$, and $\mathrm{pKa}$ which determines solubility of the unionized state of the drug molecule.

FSM and $\mathrm{RB}$ are potent $5-\mathrm{HT}_{1 \mathrm{~B} / \mathrm{D}}$ receptor agonists and have molecular weights of $379.41 \mathrm{~g} / \mathrm{mol}$ and $391.47 \mathrm{~g} / \mathrm{mol}$, respectively. Pharmacological studies have demonstrated that FSM is cerebroselective [57]. FSM and RB are good TDD candidates because of their small molecular weight, lipophilicity, partition coefficient and solubility.
Only few compounds can traverse the skin in sufficient quantity to provide a therapeutic effect. FSM has a long terminal elimination halflife time of 26 hours compared to other triptans (RB half-life is 2-3 hours). Evers et al investigated the efficacy of oral FSM as compared to other triptans in migraine with aura through a metaanalysis study [57]. Their data confirmed that FSM provided an efficacious treatment for migraine attacks with aura when taken during the headache phase and had significantly lower relapse rate as compared to rizatriptan, zolmitriptan, and almotriptan(57).

In our study, FSM had a flux value of $25.7 \mu \mathrm{g} / \mathrm{cm}^{2} / \mathrm{h}$ when microneedle rollers were used on porcine skin in comparison to passive diffusion $\left(13.4 \mu \mathrm{g} / \mathrm{cm}^{2} / \mathrm{h}\right)$. RB had a microneedle-mediated flux rate of $51.8 \mu \mathrm{g} / \mathrm{cm}^{2} / \mathrm{h}$ and a passive flux rate of $20.2 \mu \mathrm{g} / \mathrm{cm}^{2} / \mathrm{h}$. The drugs had enhanced transport rates across microneedle-mediated skin in comparison with passive diffusion. Microconduits created by the microneedle rollers led to higher transdermal flux values. Microneedle rollers significantly increased the percutaneous transport of both frovatriptan succinate monohydrate and rizatriptan benzoate across pig ear skin in vitro. 
Though there are several reports using microneedles for transdermal drug delivery, there are still many hurdles for scientists and researchers to cross. In summary, the results of our study provide evidence that solid microneedle rollers can be used to deliver antimigraine drugs, FSM and RB in vitro across the skin.

\section{Conclusion}

The use of a microneedle roller led to a statistically significant difference in transdermal flux values of FSM and RB in comparison to passive delivery. There was an increase in flux values for medications when microneedles were used, in contrast to passive diffusion. In our study, it was demonstrated that the microneedle device disrupted the SC leading to enhanced delivery of FSM and RB. Future studies can investigate the usefulness of other types of microneedles such as coated, dissolving, hollow, or hydrogel-forming microneedles for the transport of these medications, as well as the use of physical and chemical enhancers.

\section{Acknowledgement}

I am grateful to Patti Edwards of the Laura Van Winkle Laboratory at UC Davis for her support with obtaining and visualizing confocal microscopy images.

\section{Author Contributions}

This study was designed by Dr. Kevin B. Ita; Diffusion studies and data analysis was performed by Gagandeep K. Malhi, under the direct supervision and guidance of Dr. Kevin B. Ita. LC-MS analysis was carried out by Dr. Inna E. Popova; LC-MS equipment was provided by Dr. Matthew J. Morra.

\section{Conflict of Interest}

The authors have no conflicts of interest to declare.

\section{References}

1. Xu H, Han W, Wang J, Li M (2016) Network meta-analysis of migraine disorder treatment by NSAIDs and triptans. J Headache Pain 17: 113. [Crossref]

2. Lipton RB, Diamond S, Reed M, Diamond ML, Stewart WF (2001) Migraine diagnosis and treatment: results from the American Migraine Study II. Headache 41: 638-645. [Crossref]

3. Ingledue VF, Mounsey A (2014) PURLs: treating migraine: the case for aspirin. J Fam Pract 63: 94-96. [Crossref]

4. Cady RK, Schreiber CP, Farmer KU (2004) Understanding the patient with migraine: the evolution from episodic headache to chronic neurologic disease. A proposed classification of patients with headache. Headache 44: 426-435.

5. Steiner TJ, Birbeck GL, Jensen R, Katsarava Z, Martelletti P, et al. (2011) The Global Campaign, World Health Organization and Lifting The Burden: collaboration in action. $J$ Headache Pain 12: 273-274. [Crossref]

6. Bloudek LM, Stokes M, Buse DC, Wilcox TK, Lipton RB, et al. (2012) Cost of healthcare for patients with migraine in five European countries: results from the International Burden of Migraine Study (IBMS). J Headache Pain 13: 361-378. [Crossref]

7. Steiner TJ, Birbeck GL, Jensen RH, Katsarava Z, Stovner LJ (2015) Headache disorders are third cause of disability worldwide. J Headache Pain 16: 58. [Crossref]

8. Sanford M (2012) Frovatriptan: a review of its use in the acute treatment of migraine. CNS Drugs 26: 791-811. [Crossref]

9. Iljazi A, Ayata C, Ashina M, Hougaard A (2018) The Role of Endothelin in the Pathophysiology of Migraine-a Systematic Review. Curr Pain Headache Rep 22: 27. [Crossref]

10. Kim DW, Lee SK (2017) Headache and Epilepsy. J Epilepsy Res 7: 7-15. [Crossref]
11. Silva-Néto RP, Almeida KJ, Bernardino SN (2014) Analysis of the duration of migraine prophylaxis. J Neurol Sci 337: 38-41. [Crossref]

12. Jay GW, Barkin RL (2017) Primary Headache Disorders Part I- Migraine and the Trigeminal Autonomic Cephalalgias. Dis Mon 2017.

13. Kim J, Cho SJ, Kim WJ, Yang KI, Yun CH, Chu MK (2017) Insomnia in tension-type headache: a population-based study. J Headache Pain 18: 95.

14. Cady RK, Farmer K (2016) Managing migraine by patient profile: role of frovatriptan. Patient Prefer Adherence 10: 501-510. [Crossref]

15. Pithadia AB, Jain SM (2009) 5-Hydroxytryptamine Receptor Subtypes and their Modulators with Therapeutic Potentials. J Clin Med Res 1: 72-80.

16. https://pubchem.ncbi.nlm.nih.gov/compound/Frovatriptan - section=Mechanism-ofAction

17. Ramachandran R (2018) Neurogenic inflammation and its role in migraine. Semin Immunopathol 40: 301-314. [Crossref]

18. Hamel E (2007) Serotonin and migraine: biology and clinical implications. Cephalalgia 27: 1293-1300. [Crossref]

19. Antonaci F, Ghiotto N, Wu S, Pucci E, Costa A (2016) Recent advances in migraine therapy. Springerplus 5: 637. [Crossref]

20. Negro A, Koverech A, Martelletti P (2018) Serotonin receptor agonists in the acute treatment of migraine: a review on their therapeutic potential. J Pain Res 11: 515-526.

21. Barnes NM, Sharp T (1999) A review of central 5-HT receptors and their function. Neuropharmacology 38: 1083-1152. [Crossref]

22. Humphrey PP, Feniuk W (1991) Mode of action of the anti-migraine drug sumatriptan Trends Pharmacol Sci 12: 444-446. [Crossref]

23. Allais G, Benedetto C (2016) Spotlight on frovatriptan: a review of its efficacy in the treatment of migraine. Drug Des Devel Ther 10: 3225-3236. [Crossref]

24. Mennini N, Orlandini S, Furlanetto S, Pasquini B, Mura P (2018) Development and Optimization by Quality by Design Strategies of Frovatriptan Orally Disintegrating Tablets for Migraine Management. Curr Drug Deliv 15: 436-445.

25. Kieu T (2002) Frovatriptan (Frova): A new Triptan. PharmaNote 18:10.

26. Silberstein SD (2002) Pharmacological profile and clinical characteristics of frovatriptan in the acute treatment of migraine: introduction. Headache 42: 45-46.

27. Williamson DJ, Hill RG, Shepheard SL, Hargreaves RJ (2001) The anti-migraine 5-HT(1B/1D) agonist rizatriptan inhibits neurogenic dural vasodilation in anaesthetized guinea-pigs. Br J Pharmacol 133: 1029-1034.

28. Anselmo AC, Mitragotri S (2014) An overview of clinical and commercial impact of drug delivery systems. J Control Release 190: 15-28.

29. Brambilla D, Luciani P, Leroux JC (2014) Breakthrough discoveries in drug delivery technologies: the next 30 years. $J$ Control Release 190: 9-14.

30. Klemp Gjertsen M, Lyngstadaas A (2007) NIPH Systematic Reviews: Executive Summaries. Efficacy and Safety of 5HT1B/D Reseptoragonists, Triptans, for the Treatment of Migraine Disorders. Oslo, Norway: Knowledge Centre for the Health Services at The Norwegian Institute of Public Health (NIPH).

31. Mayans L, Walling A (2018) Acute Migraine Headache: Treatment Strategies American family physician 97: 243-251.

32. Schoellhammer CM, Blankschtein D, Langer R (2014) Skin permeabilization for transdermal drug delivery: recent advances and future prospects. Expert Opin Drug Deliv 11: 393-407. [Crossref]

33. Paudel KS, Milewski M, Swadley CL, Brogden NK, Ghosh P (2010) Challenges and opportunities in dermal/transdermal delivery. Ther Deliv 1: 109-131. [Crossref]

34. Han T, Das DB (2015) Potential of combined ultrasound and microneedles for enhanced transdermal drug permeation: a review. Eur J Pharm Biopharm 89: 312-328.

35. Naik A, Kalia YN, Guy RH (2000) Transdermal drug delivery: overcoming the skin's barrier function. Pharm Sci Technolo Today 3: 318-326.

36. Al-Zahrani S, Zaric M, McCrudden C, Scott C, Kissenpfennig A, et al. (2012) Microneedle-mediated vaccine delivery: harnessing cutaneous immunobiology to improve efficacy. Expert Opin Drug Deliv 9: 541-550.

37. Kretsos K, Kasting GB (2007) A geometrical model of dermal capillary clearance. Math Biosci 208: 430-453. 
38. Donnelly RF, Singh TR, Garland MJ, Migalska K, Majithiya R, et al. (2012) HydrogelForming Microneedle Arrays for Enhanced Transdermal Drug Delivery. Adv Funct Mater 22: 4879-4890.

39. Arora A, Prausnitz MR, Mitragotri S (2008) Micro-scale devices for transdermal drug delivery. Int J Pharm 364: 227-236. [Crossref]

40. Tuan-Mahmood TM, McCrudden MT, Torrisi BM, McAlister E, Garland MJ, et al. (2013) Microneedles for intradermal and transdermal drug delivery. Eur J Pharm Sci 50: 623-637.

41. Prausnitz MR1, Langer R (2008) Transdermal drug delivery. Nat Biotechnol 26: 12611268. [Crossref]

42. Godin B, Touitou E (2007) Transdermal skin delivery: predictions for humans from in vivo, ex vivo and animal models. Adv Drug Deliv Rev 59: 1152-1161. [Crossref]

43. Van Gele M, Geusens B, Brochez L, Speeckaert R, Lambert J (2011) Three-dimensional skin models as tools for transdermal drug delivery: challenges and limitations. Expert Opin Drug Deliv 8: 705-720.

44. Garcia MTJ, Vasconcelos FLL, Raffier CP, Roberts MS, Grice JE, et al. (2018) Alternative methods to animal studies for the evaluation of topical/transdermal drug delivery systems. Curr Top Med Chem 18: 287-299.

45. Karadzovska D, Brooks JD, Monteiro-Riviere NA, Riviere JE (2013) Predicting skin permeability from complex vehicles. Adv Drug Deliv Rev 65: 265-277. [Crossref]

46. Jepps OG, Dancik Y, Anissimov YG, Roberts MS (2013) Modeling the human skin barrier--towards a better understanding of dermal absorption. Adv Drug Deliv Rev 65: 152-168. [Crossref]

47. Zorec B, Zupancic S, Kristl J, Pavselj N (2018) Combinations of nanovesicles and physical methods for enhanced transdermal delivery of a model hydrophilic drug. Eur J Pharm Biopharm 127: 387-397.

48. Puri A, Murnane KS, Blough BE, Banga AK (2017) Effects of chemical and physical enhancement techniques on transdermal delivery of 3-fluoroamphetamine hydrochloride. Int J Pharm 528: 452-462.
49. Kaur M, Ita KB, Popova IE, Parikh SJ, Bair DA (2014) Microneedle-assisted delivery of verapamil hydrochloride and amlodipine besylate. Eur J Pharm Biopharm 86: 284-291.

50. Bal SM, Kruithof AC, Zwier R, Dietz E, Bouwstra JA, et al. (2010) Influence of microneedle shape on the transport of a fluorescent dye into human skin in vivo. $J$ Control Release 147: 218-224.

51. Alkilani AZ, McCrudden MT, Donnelly RF (2015) Transdermal Drug Delivery: Innovative Pharmaceutical Developments Based on Disruption of the Barrier Properties of the stratum corneum. Pharma 7: 438-470.

52. Hao Y, Li W, Zhou X, Yang F, Qian Z (2017) Microneedles-Based Transdermal Drug Delivery Systems: A Review. J Biomed Nanotechnol 13: 1581-1597.

53. Vitorino C, Almeida A, Sousa J, Lamarche I, Gobin P, et al. (2014) Passive and active strategies for transdermal delivery using co-encapsulating nanostructured lipid carriers: In vitro vs. in vivo studies. Eur J Pharm Biopharm 86: 133-144.

54. Hoang MT, Ita KB, Bair DA (2015) Solid Microneedles for Transdermal Delivery of Amantadine Hydrochloride and Pramipexole Dihydrochloride. Pharma 7: 379-396.

55. Bhatt RH, Micali G, Galinkin J, Palicharla P, Koch RL, et al. (1997) Determination and correlation of in vitro viability for hairless mouse and human neonatal whole skin and stratum corneum/epidermis. Arch Dermatol Res 289: 170-173.

56. Hikima T, Tamura Y, Yamawaki Y, Yamamoto M, Tojo K (2013) Skin accumulation and penetration of a hydrophilic compound by a novel gemini surfactant, sodium dilauramidoglutamide lysine. Int J Pharm 443: 288-292. [Crossref]

57. Evers S, Savi L, Omboni S, Lisotto C, Zanchin G (2015) Efficacy of frovatriptan a compared to other triptans in migraine with aura. J Headache Pain 16: 514. [Crossref]

58. Yan G, Warner KS, Zhang J, Sharma S, Gale BK (2010) Evaluation needle length and density of microneedle arrays in the pretreatment of skin for transdermal drug delivery. Int J pharma 391:7-12.

Copyright: (C)2018 Malhi GK. This is an open-access article distributed under the terms of the Creative Commons Attribution License, which permits unrestricted use, distribution, and reproduction in any medium, provided the original author and source are credited. 